\title{
One Raised Product Prime Labeling of Some Star Related Graphs
}

\author{
B S Sunoj ${ }^{1 *}$, T K Mathew Varkey ${ }^{2}$ \\ ${ }^{1}$ Department of Mathematics, Government Polytechnic College, Attingal, Kerala, India \\ ${ }^{2}$ Department of Mathematics, TKM College of Engineering, Kollam, Kerala, India \\ *Corresponding Author: spalazhi@yahoo.com, Tel.: +91-9495257203
}

Available online at: www.isroset.org

Received: 9/May/2018, Revised: 23/May/2018, Accepted: 18/Jun/2018, Online: 30/Jun/2018

\begin{abstract}
One raised product prime labeling of a simple. finite and un direct graph $\mathrm{G}$ with p vertices and q edges is the labeling of the vertices of the graph with first $\mathrm{p}$ natural numbers, edges with product of the labels of the end vertices plus one. If the greatest common divisor of each vertex of degree greater than one is one then the graph is called one raised product prime graph. Here the greatest common divisor of the labels of the edges incident on a vertex is called greatest common incidence number of that vertex. Here we proved that star, bistar, splitting graph of a star, shadow graph of a star, jelly fish graph and some more star related graphs admit one raised product prime labeling.
\end{abstract}

Keywords - Graph labeling, product, prime labeling, prime graphs, star

\section{INTRODUCTION}

The concept of one raised product prime labeling was introduced in [5] by Sunoj B S and Mathew Varkey T K. Later in [6] and [7] they extend the study and proved the result for some path related graphs and some snake graphs. In this paper we proved that star, bistar, graph obtained by duplicating the apex vertex of star by a vertex, graph obtained by duplicating an edge of a star by an edge, graph obtained by duplicating the apex vertex of star by an edge, splitting graph of a star, shadow graph of a star, jelly fish graph and tensor product of star with path of length one admit one raised product prime labeling.

The paper contains three sections in which section I contains introduction to one raised product prime labeling, section II contains preliminaries and notations and section III contains main results and illustrations and section IV contains concluding remarks.

\section{Preliminaries And Notations}

In this paper we consider only those graphs which are simple finite and undirected. Graph is denoted by G, vertex set is denoted by $\mathrm{V}$, edge set is denoted by $\mathrm{E}, \mathrm{p}$ is the number of vertices of the graph $\mathrm{G}$ and $\mathrm{q}$ is the number of edges of the graph G. $|\mathrm{V}(\mathrm{G})|=$ number of vertices of graph $\mathrm{G},|\mathrm{E}(\mathrm{G})|=$ number of edges of graph $\mathrm{G}$.

Definition 2.1[3] If the vertices and edges of the graph are assigned values subject to certain conditions is known as graph labeling.
Definition 2.2[8] The greatest common incidence number of a vertex is define as the greatest common divisor [1] of the labels of the edges incident on that vertex.

Definition 2.3[5] Let $\mathrm{G}=(\mathrm{V}(\mathrm{G}), \mathrm{E}(\mathrm{G}))$ be a graph with $\mathrm{p}$ vertices and q edges . Define a bijection

$\mathrm{f}: \mathrm{V}(\mathrm{G}) \rightarrow\{1,2,---, \mathrm{p}\}$ by $\mathrm{f}\left(\mathrm{v}_{\mathrm{i}}\right)=\mathrm{i}$, for every $\mathrm{i}$ from 1 to $\mathrm{p}$ and define a 1-1 mapping $f_{\text {orpp }}^{*}: \mathrm{E}(\mathrm{G}) \rightarrow$ set of natural numbers $\mathrm{N}$ by $f_{o r p p}^{*}(u v)=f(u) f(v)+1$.The induced function $f_{o r p p}^{*}$ is said to be one raised product prime labeling, if for each vertex of degree at least 2 , the gcin of the labels of the incident edges is 1.

Definition 2.4[5] A graph which admits one raised product prime labeling is called one raised product prime graph.

Definition 2.5 [2] A tree with one internal node and $n$ leaves is called star and is denoted by $K_{1, n}$.

Definition 2.6[4] For a graph $G$ the splitting graph is obtained by adding to each vertex $\mathrm{v}$ a new vertex $\mathrm{v}^{\prime}$ such that $\mathrm{v}^{\prime}$ is adjacent to every vertex that is adjacent to $\mathrm{v}$ in $\mathrm{G}$. The resultant graph is denoted as $\mathrm{S}^{\prime}(\mathrm{G})$.

Definition 2.7 [3] The shadow graph $\mathrm{D}_{2}(\mathrm{G})$ of a connected graph $G$ is constructed by taking two copies of $G$ say $G^{\prime}$ and $G^{\prime \prime}$ join each vertex $v^{\prime}$ in $G^{\prime}$ to the neighbors of the corresponding vertices in $\mathrm{G}^{\prime \prime}$.

Definition 2.8 [3] Let $G$ be a cycle of length 4 with one chord. The graph obtained by joining the apex vertex of star $\mathrm{K}_{1, \mathrm{n}}$ and star $\mathrm{K}_{1, \mathrm{~m}}$ to the vertices of degree 2 is called jelly fish graph and is denoted by $\mathrm{JF}(\mathrm{m}, \mathrm{n})$.

Definition 2.9 [3] Tensor product of path $\mathrm{P}_{2}$ with star $\mathrm{K}_{1, \mathrm{n}}$ is two copies of star $K_{1, n+1}$. 
Definition 2.10 [3] Duplication of a vertex $v$ by a new vertex of graph $\mathrm{G}$ produces a new graph $\mathrm{H}$ by adding a vertex $\mathrm{u}$ and joining $\mathrm{u}$ to vertices of $\mathrm{G}$ adjacent to $\mathrm{v}$.

Definition 2.11 [3] Duplication of a vertex $v$ by a new edge of graph $\mathrm{G}$ produces a new graph $\mathrm{H}$ by adding an edge and joining the end vertices of the edge to $\mathrm{v}$.

Definition 2.12 [3] Duplication of an edge $\mathbf{e}(=\mathbf{a b})$ by a new edge $\mathbf{f}(=\mathbf{u v})$ of graph $\mathrm{G}$ produces a new graph $\mathrm{H}$ by adding an edge $\mathbf{f}$ and joining $\mathbf{u}$ to the neighboring vertices of $\mathbf{a}$ other than $\mathbf{b}$ and joining $\mathbf{v}$ to the neighboring vertices of $\mathbf{b}$ other than $\mathbf{a}$.

\section{MAIN RESUltS}

Theorem 3.1 Star $\mathrm{K}_{1, \mathrm{n}}(\mathrm{n}>2)$ admits one raised product prime labeling.

Proof: Let $\mathrm{G}=\mathrm{K}_{1, \mathrm{n}}$ and let $\mathrm{v}_{1}, \mathrm{v}_{2},---, \mathrm{v}_{\mathrm{n}+1}$ are the vertices of $\mathrm{G}$. Here $|\mathrm{V}(\mathrm{G})|=\mathrm{n}+1$ and $|\mathrm{E}(\mathrm{G})|=\mathrm{n}$.

Define a function $\mathrm{f}: \mathrm{V} \rightarrow\{1,2,---, \mathrm{n}+1\}$ by

Clearly $f$ is a bijection.

$$
f\left(v_{i}\right)=i, i=1,2,---, n+1
$$

For the vertex labeling $\mathrm{f}$, the induced edge labeling $f_{\text {orpp }}^{*}$ is defined as follows

$f_{\text {orpp }}^{*}\left(v_{1} v_{i+1}\right) \quad=\mathrm{i}+2, \quad \mathrm{i}=1,2,---, \mathrm{n}$.

Clearly $f_{\text {orpp }}^{*}$ is an injection.

$\operatorname{gcin}$ of $\left(\mathrm{v}_{1}\right)=\operatorname{gcd}$ of $\left\{f_{\text {orpp }}^{*}\left(v_{1} v_{2}\right), f_{\text {orpp }}^{*}\left(v_{1} v_{3}\right)\right\}$

$$
=\operatorname{gcd} \text { of }\{3,4\}=1 \text {. }
$$

So, gcin of each vertex of degree greater than one is 1 .

Hence $\mathrm{K}_{1, \mathrm{n}}$, admits one raised product prime labeling.

Example 3.1 $\mathrm{G}=\mathrm{K}_{1,4}$

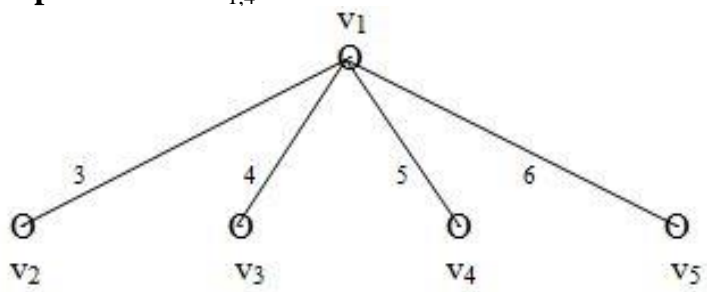

fig -3.1

Theorem 3.2 Bistar B(m,n) ( $m$ and $n>2$ ) admits one raised product prime labeling.

Proof: Let $\mathrm{G}=\mathrm{B}(\mathrm{m}, \mathrm{n})$ and let $\mathrm{v}_{1}, \mathrm{v}_{2},---, \mathrm{v}_{\mathrm{m}+\mathrm{n}+2}$ are the vertices of G.

Here $|\mathrm{V}(\mathrm{G})|=m+n+2$ and $|\mathrm{E}(\mathrm{G})|=m+n+1$.

Define a function $\mathrm{f}: \mathrm{V} \rightarrow\{1,2,---, \mathrm{m}+\mathrm{n}+2\}$ by

Clearly $f$ is a bijection.

$$
\mathrm{f}\left(\mathrm{v}_{\mathrm{i}}\right)=\mathrm{i}, \mathrm{i}=1,2,---\mathrm{m}+\mathrm{n}+2
$$

For the vertex labeling $\mathrm{f}$, the induced edge labeling $f_{\text {orpp }}^{*}$ is defined as follows

$$
\begin{array}{ll}
f_{\text {orpp }}^{*}\left(v_{m+1} v_{i}\right) & =\mathrm{mi}+\mathrm{i}+1, \quad \mathrm{i}=1,2,---\mathrm{m} . \\
f_{\text {orpp }}^{*}\left(v_{m+1} v_{m+2}\right) & =\mathrm{m}^{2}+3 \mathrm{~m}+3 . \\
f_{\text {orpp }}^{*}\left(v_{m+2} v_{m+i+2}\right) & =(\mathrm{m}+2)^{2}+\mathrm{i}(\mathrm{m}+2)+1,
\end{array}
$$

Clearly $f_{o r p p}^{*}$ is an injection.

$$
\text { gcin of }\left(\mathrm{v}_{\mathrm{m}+1}\right)
$$$$
=\operatorname{gcd} \text { of }\left\{f_{o r p p}^{*}\left(v_{m} v_{m+1}\right)\right. \text {, }
$$$$
\left.f_{\text {orpp }}^{*}\left(v_{m+1} v_{m+2}\right)\right\}
$$$$
=\operatorname{gcd} \text { of }\left\{\mathrm{m}^{2}+\mathrm{m}+1, \mathrm{~m}^{2}+3 \mathrm{~m}+3\right\}
$$$$
=\operatorname{gcd} \text { of }\left\{2 \mathrm{~m}+2, \mathrm{~m}^{2}+\mathrm{m}+1\right\}
$$$$
=\operatorname{gcd} \text { of }\{\mathrm{m}+1, \mathrm{~m}(\mathrm{~m}+1)+1\}
$$$$
=1 \text {. }
$$

$\operatorname{gcin}$ of $\left(\mathrm{v}_{\mathrm{m}+2}\right)$

$$
\begin{aligned}
= & \operatorname{gcd} \text { of }\left\{f_{\text {orpp }}^{*}\left(v_{m+1} v_{m+2}\right),\right. \\
& \left.\quad f_{\text {orpp }}^{*}\left(v_{m+2} v_{m+3}\right)\right\} \\
= & \operatorname{gcd} \text { of }\left\{\mathrm{m}^{2}+3 \mathrm{~m}+3, \mathrm{~m}^{2}+5 \mathrm{~m}+7\right\} \\
= & \operatorname{gcd} \text { of }\left\{2 \mathrm{~m}+4, \mathrm{~m}^{2}+3 \mathrm{~m}+3\right\} \\
= & \operatorname{gcd} \text { of }\{\mathrm{m}+2,(\mathrm{~m}+1)(\mathrm{m}+2)+1\} \\
= & 1 .
\end{aligned}
$$

So, gcin of each vertex of degree greater than one is 1 . Hence $B(m, n)$, admits one raised product prime labeling.

Example 3.2 $\mathrm{G}=\mathrm{B}(4,3)$

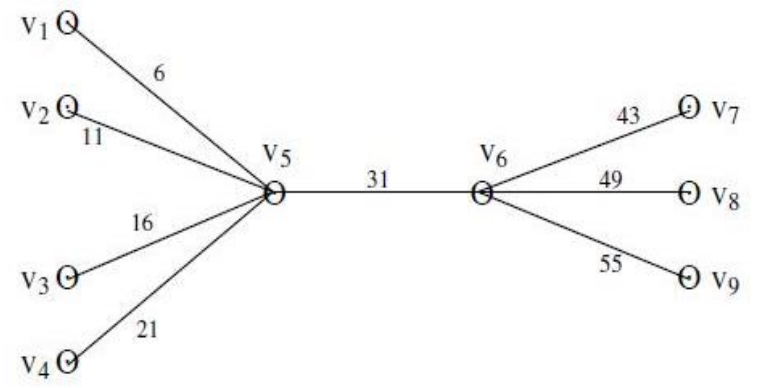

fig -3.2

Theorem 3.3 Let $\mathrm{G}$ be the graph obtained by duplicating the apex vertex of star $K_{1, n}(n>2)$. $G$ admits one raised product prime labeling.

Proof: Let $\mathrm{G}$ be the graph and let $\mathrm{v}_{1}, \mathrm{v}_{2},---, \mathrm{v}_{\mathrm{n}+2}$ are the vertices of $\mathrm{G}$.

Here $|\mathrm{V}(\mathrm{G})|=\mathrm{n}+2$ and $|\mathrm{E}(\mathrm{G})|=2 \mathrm{n}$.

Define a function $\mathrm{f}: \mathrm{V} \rightarrow\{1,2,---, \mathrm{n}+2\}$ by

Clearly $\mathrm{f}$ is a bijection.

$$
f\left(v_{i}\right)=i, i=1,2,---, n+2
$$

For the vertex labeling $\mathrm{f}$, the induced edge labeling $f_{\text {orpp }}^{*}$ is defined as follows

$$
\begin{array}{lll}
f_{\text {orpp }}^{*}\left(v_{i} v_{n+1}\right) & =\mathrm{ni}+\mathrm{i}+1, & \mathrm{i}=1,2,---, \mathrm{n} . \\
f_{\text {orpp }}^{*}\left(v_{i} v_{n+2}\right) & =\mathrm{ni}+2 \mathrm{i}+1, & \mathrm{i}=1,2,---, \mathrm{n} .
\end{array}
$$

Clearly $f_{\text {orpp }}^{*}$ is an injection.

$$
\begin{aligned}
\operatorname{gcin} \text { of }\left(\mathrm{v}_{\mathrm{i}}\right) & \operatorname{gcd} \text { of }\left\{f_{\text {orpp }}^{*}\left(v_{i} v_{n+1}\right),\right. \\
& \left.\quad f_{\text {orpp }}^{*}\left(v_{i} v_{n+2}\right)\right\} \\
= & \operatorname{gcd} \text { of }\{\mathrm{ni}+\mathrm{i}+1, \mathrm{ni}+2 \mathrm{i}+1\} \\
= & 1, \quad \operatorname{icd} \text { of }\{\mathrm{i},(\mathrm{n}+1) \mathrm{i}+1\} \\
= & \operatorname{gcd} \text { of }\left\{f_{\text {orpp }}^{*}\left(v_{1} v_{n+1}\right),\right. \\
\operatorname{gcin} \text { of }\left(\mathrm{v}_{\mathrm{n}+1}\right) & \left.\quad \quad_{\text {orpp }}^{*}\left(v_{2} v_{n+1}\right)\right\} \\
= & \operatorname{gcd} \text { of }\{\mathrm{n}+2,2 \mathrm{n}+3\} \\
= & \operatorname{gcd} \text { of }\{\mathrm{n}+1, \mathrm{n}+2\} \\
= & \operatorname{gcd} \text { of }\left\{f_{\text {orpp }}^{*}\left(v_{1} v_{n+2}\right),\right. \\
\operatorname{gcin} \text { of }\left(\mathrm{v}_{\mathrm{n}+2}\right) \quad & \left.\quad f_{\text {orpp }}^{*}\left(v_{2} v_{n+2}\right)\right\} \\
& \operatorname{gcd} \text { of }\{\mathrm{n}+3,2 \mathrm{n}+5\}
\end{aligned}
$$




$$
\begin{aligned}
& =\operatorname{gcd} \text { of }\{n+2, n+3\} \\
& =1 .
\end{aligned}
$$

So, gcin of each vertex of degree greater than one is 1 . Hence $\mathrm{G}$, admits one raised product prime labeling.

Example 3.3 Let $\mathrm{G}$ be the graph obtained by duplicating the apex vertex of star $\mathrm{K}_{1,4}$

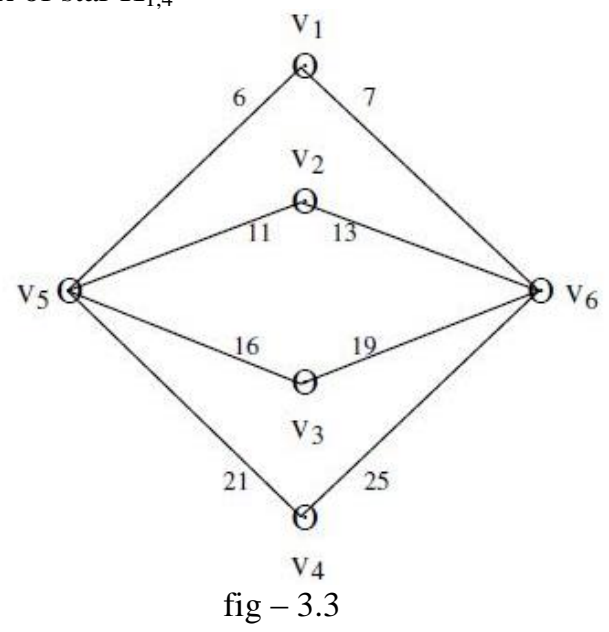

Theorem 3.4 Let $\mathrm{G}$ be the graph obtained by duplicating an edge of star $K_{1, n}$ ( $n$ is a natural number greater than 2). $G$ admits one raised product prime labeling.

Proof: Let $\mathrm{G}$ be the graph and let $\mathrm{v}_{1}, \mathrm{v}_{2},---, \mathrm{v}_{\mathrm{n}+3}$ are the vertices of $\mathrm{G}$.

Here $|V(G)|=n+3$ and $|E(G)|=2 n+1$.

Define a function $\mathrm{f}: \mathrm{V} \rightarrow\{1,2,---, \mathrm{n}+3\}$ by

Clearly $f$ is a bijection.

$$
f\left(v_{i}\right)=i, i=1,2,---, n+3
$$

For the vertex labeling $\mathrm{f}$, the induced edge labeling $f_{o r p p}^{*}$ is defined as follows

$$
\begin{aligned}
& f_{\text {orpp }}^{*}\left(v_{i} v_{n+1}\right) \quad=\mathrm{ni}+\mathrm{i}+1, \quad \mathrm{i}=1,2,---, \mathrm{n} \text {. } \\
& f_{\text {orpp }}^{*}\left(v_{i} v_{n+2}\right) \quad=\mathrm{ni}+2 \mathrm{i}+1, \quad \mathrm{i}=1,2,---, \mathrm{n} \text {. } \\
& f_{\text {orpp }}^{*}\left(v_{n+2} v_{n+3}\right) \quad=\mathrm{n}^{2}+5 \mathrm{n}+7 \text {. } \\
& \text { Clearly } f_{\text {orpp }}^{*} \text { is an injection. } \\
& \operatorname{gcin} \text { of }\left(\mathrm{v}_{\mathrm{i}}\right)=\operatorname{gcd} \text { of }\left\{f_{\text {orpp }}^{*}\left(v_{i} v_{n+1}\right)\right. \text {, } \\
& =1, \quad \mathrm{i}=1,2,---, \mathrm{n} \text {. } \\
& \operatorname{gcin} \text { of }\left(\mathrm{v}_{\mathrm{n}+1}\right)=\operatorname{gcd} \text { of }\left\{f_{\text {orpp }}^{*}\left(v_{1} v_{n+1}\right)\right. \text {, } \\
& =1 \text {. } \\
& \left.f_{o r p p}^{*}\left(v_{2} v_{n+1}\right)\right\} \\
& \operatorname{gcin} \text { of }\left(\mathrm{v}_{\mathrm{n}+2}\right)=\operatorname{gcd} \text { of }\left\{f_{\text {orpp }}^{*}\left(v_{1} v_{n+2}\right)\right. \text {, } \\
& \left.f_{o r p p}^{*}\left(v_{2} v_{n+2}\right)\right\} \\
& =1 \text {. }
\end{aligned}
$$

So, gcin of each vertex of degree greater than one is 1 . Hence $\mathrm{G}$, admits one raised product prime labeling.

Example 3.4 Let $\mathrm{G}$ be the graph obtained by duplicating an edge of star $\mathrm{K}_{1,4}$

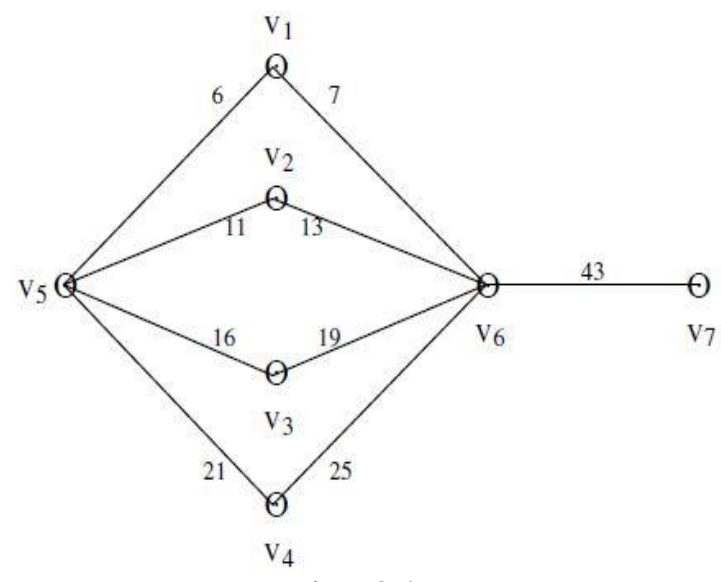

Fig -3.4

Theorem 3.5 Let $\mathrm{G}$ be the graph obtained by duplicating the apex vertex of star $K_{1, n}(n>2)$ by an edge. $G$ admits one raised product prime labeling.

Proof: Let $\mathrm{G}$ be the graph and let $\mathrm{v}_{1}, \mathrm{v}_{2},---, \mathrm{v}_{\mathrm{n}+3}$ are the vertices of $\mathrm{G}$.

Here $|\mathrm{V}(\mathrm{G})|=\mathrm{n}+3$ and $|\mathrm{E}(\mathrm{G})|=\mathrm{n}+3$.

Define a function $\mathrm{f}: \mathrm{V} \rightarrow\{1,2,---, \mathrm{n}+3\}$ by

$$
f\left(v_{i}\right)=i, i=1,2,---, n+3
$$

Clearly $f$ is a bijection.

For the vertex labeling $\mathrm{f}$, the induced edge labeling $f_{\text {orpp }}^{*}$ is defined as follows

$$
\begin{array}{rlr}
f_{\text {orpp }}^{*}\left(v_{1} v_{2}\right) & =3 . & \\
f_{\text {orpp }}^{*}\left(v_{1} v_{3}\right) & =4 & \\
f_{\text {orpp }}^{*}\left(v_{2} v_{3}\right) & =7 . & \\
f_{\text {orpp }}^{*}\left(v_{3} v_{i+3}\right) & =3 \mathrm{i}+10, & \mathrm{i}=1,2,---, \mathrm{n} .
\end{array}
$$

Clearly $f_{\text {orpp }}^{*}$ is an injection.

$$
\begin{aligned}
\operatorname{gcin} \text { of }\left(\mathrm{v}_{1}\right) \quad & \operatorname{gcd} \text { of }\left\{f_{\text {orpp }}^{*}\left(v_{1} v_{2}\right),\right. \\
& \left.f_{\text {orpp }}^{*}\left(v_{1} v_{3}\right)\right\} \\
= & \operatorname{gcd} \text { of }\{3,4\} \\
= & 1 . \\
= & \operatorname{gcd} \text { of }\left\{f_{\text {orpp }}^{*}\left(v_{1} v_{2}\right),\right. \\
\operatorname{gcin} \text { of }\left(\mathrm{v}_{2}\right) \quad & \left.f_{\text {orpp }}^{*}\left(v_{2} v_{3}\right)\right\} \\
= & \operatorname{gcd} \text { of }\{3,7\} \\
= & 1 . \\
= & \operatorname{gcd} \text { of }\left\{f_{\text {orpp }}^{*}\left(v_{1} v_{3}\right),\right. \\
\operatorname{gcin} \text { of }\left(\mathrm{v}_{3}\right) \quad & \left.f_{\text {orpp }}^{*}\left(v_{2} v_{3}\right)\right\} \\
= & \operatorname{gcd} \text { of }\{4,7\} \\
= & 1 .
\end{aligned}
$$

So, gcin of each vertex of degree greater than one is 1 . Hence $\mathrm{G}$, admits one raised product prime labeling. Example 3.5 Let $\mathrm{G}$ be the graph obtained by duplicating the apex vertex of star $\mathrm{K}_{1,4}$ 


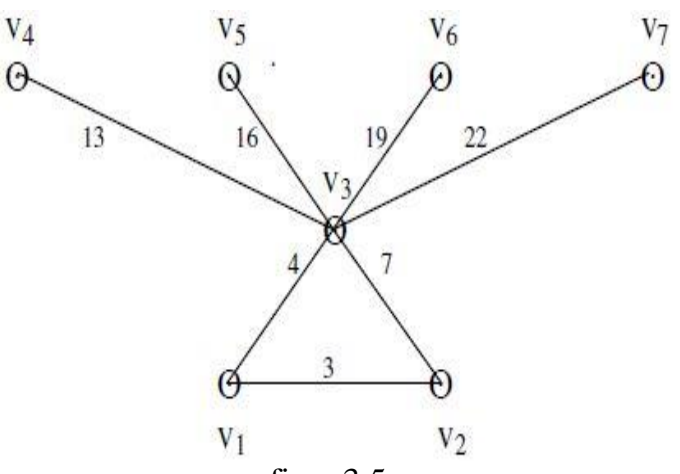

fig -3.5

Theorem 3.6 Splitting graph of star $K_{1, n}(n>2)$ admits one raised product prime labeling.

Proof: Let $\mathrm{G}=\mathrm{S}^{\prime}\left(\mathrm{K}_{1, \mathrm{n}}\right)$ be the graph and let $\mathrm{v}_{1}, \mathrm{v}_{2},---, \mathrm{v}_{2 \mathrm{n}+2}$ are the vertices of $\mathrm{G}$.

Here $|\mathrm{V}(\mathrm{G})|=2 \mathrm{n}+2$ and $|\mathrm{E}(\mathrm{G})|=3 \mathrm{n}$.

Define a function $\mathrm{f}: \mathrm{V} \rightarrow\{1,2,---, 2 \mathrm{n}+2\}$ by

Clearly $\mathrm{f}$ is a bijection.

$$
f\left(v_{i}\right)=i, i=1,2,--, 2 n+2
$$

For the vertex labeling $\mathrm{f}$, the induced edge labeling $f_{\text {orpp }}^{*}$ is defined as follows

$$
\begin{array}{llr}
f_{\text {orp }}^{*}\left(v_{i} v_{n+1}\right) & =\mathrm{in}+\mathrm{i}+1, & \mathrm{i}=1,2,---, \mathrm{n} . \\
f_{\text {orpp }}^{*}\left(v_{i} v_{n+2}\right) & =\mathrm{in}+2 \mathrm{i}+1, \quad \mathrm{i}=1,2,---, \mathrm{n} . \\
f_{\text {orpp }}^{*}\left(v_{n+2} v_{n+2+i}\right) & =(\mathrm{n}+2)^{2}+\mathrm{i}(\mathrm{n}+2)+1, \\
& & \mathrm{i}=1,2,---, \mathrm{n} .
\end{array}
$$

Clearly $f_{\text {orpp }}^{*}$ is an injection.

$$
\begin{aligned}
\operatorname{gcin} \text { of }\left(\mathrm{v}_{\mathrm{i}}\right) & \operatorname{gcd} \text { of }\left\{f_{\text {orpp }}^{*}\left(v_{i} v_{n+1}\right),\right. \\
& \left.\quad f_{\text {orpp }}^{*}\left(v_{i} v_{n+2}\right)\right\} \\
= & \operatorname{gcd} \text { of }\{\mathrm{ni}+\mathrm{i}+1, \mathrm{ni}+2 \mathrm{i}+1\} \\
= & \operatorname{gcd}\{\mathrm{i},(\mathrm{n}+1) \mathrm{i}+1\} \\
= & \operatorname{gcd} \text { of }\left\{f_{\text {orpp }}^{*}\left(v_{1} v_{n+1}\right),\right. \\
& \left.\quad f_{\text {orpp }}^{*}\left(v_{2} v_{n+1}\right)\right\} \\
= & \operatorname{gcd} \text { of }\{\mathrm{n}+2,2 \mathrm{n}+3\} \\
= & \operatorname{gcd} \text { of }\{\mathrm{n}+1, \mathrm{n}+2\} \\
= & 1 \\
= & \operatorname{gcd} \text { of }\left\{f_{\text {orpp }}^{*}\left(v_{1} v_{n+2}\right),\right. \\
& \left.\quad f_{\text {orpp }}^{*}\left(v_{2} v_{n+2}\right)\right\} \\
= & \operatorname{gcd} \text { of }\{\mathrm{n}+3,2 \mathrm{n}+5\} \\
= & \operatorname{gcd} \text { of }\{\mathrm{n}+2, \mathrm{n}+3\} \\
= & 1
\end{aligned}
$$

So, $\boldsymbol{g c i n}$ of each vertex of degree greater than one is 1 . Hence $\mathrm{G}$, admits one raised product prime labeling.

Example 3.6 $\mathrm{G}=\mathrm{S}^{\prime}\left(\mathrm{K}_{1,4}\right)$

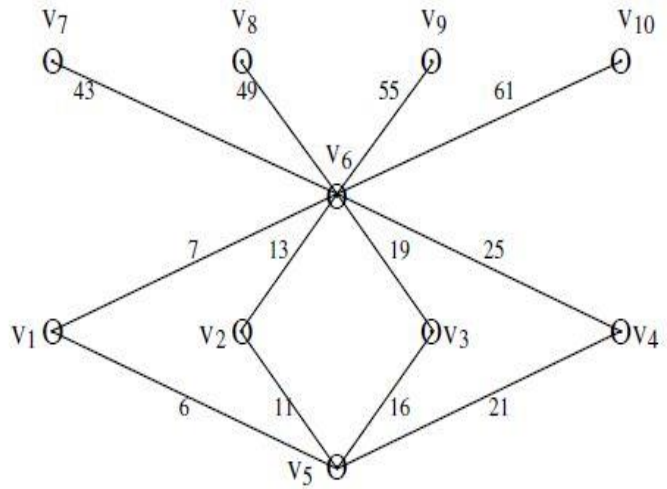

fig -3.6

Theorem 3.7 Shadow graph of star $K_{1, n}(n>2)$ admits one raised product prime labeling.

Proof: Let $\mathrm{G}=\mathrm{D}\left(\mathrm{K}_{1, \mathrm{n}}\right)$ be the graph and let $\mathrm{v}_{1}, \mathrm{v}_{2},---, \mathrm{v}_{2 \mathrm{n}+2}$ are the vertices of $\mathrm{G}$.

Here $|\mathrm{V}(\mathrm{G})|=2 \mathrm{n}+2$ and $|\mathrm{E}(\mathrm{G})|=4 \mathrm{n}$.

Define a function $\mathrm{f}: \mathrm{V} \rightarrow\{1,2,---, 2 \mathrm{n}+2\}$ by

Clearly $\mathrm{f}$ is a bijection.

$$
\mathrm{f}\left(\mathrm{v}_{\mathrm{i}}\right)=\mathrm{i}, \mathrm{i}=1,2,---, 2 \mathrm{n}+2
$$

For the vertex labeling $\mathrm{f}$, the induced edge labeling $f_{\text {orpp }}^{*}$ is defined as follows

$$
\begin{array}{lll}
f_{\text {orpp }}^{*}\left(v_{i} v_{2 n+1}\right) & =2 \mathrm{ni}+\mathrm{i}+1, \quad \mathrm{i}=1,2,---, \mathrm{n} . \\
f_{\text {orpp }}^{*}\left(v_{i} v_{2 n+2}\right) & =2 \mathrm{ni}+2 \mathrm{i}+1, \quad \mathrm{i}=1,2,---, \mathrm{n} . \\
f_{\text {orpp }}^{*}\left(v_{n+i} v_{2 n+1}\right) & =(\mathrm{n}+\mathrm{i})(2 \mathrm{n}+1)+1, \mathrm{i}=1,2,---, \mathrm{n} . \\
f_{\text {orpp }}^{*}\left(v_{n+i} v_{2 n+2}\right) & =(\mathrm{n}+\mathrm{i})(2 \mathrm{n}+2)+1, \mathrm{i}=1,2,---, \mathrm{n} .
\end{array}
$$

Clearly $f_{\text {orpp }}^{*}$ is an injection.

$$
\begin{aligned}
& \operatorname{gcin} \text { of }\left(\mathrm{v}_{\mathrm{i}}\right) \quad=\operatorname{gcd} \text { of }\left\{f_{\text {orpp }}^{*}\left(v_{i} v_{2 n+1}\right)\right. \text {, } \\
& \left.f_{\text {orpp }}^{*}\left(v_{i} v_{2 n+2}\right)\right\} \\
& =\operatorname{gcd} \text { of }\{2 \mathrm{ni}+\mathrm{i}+1,2 \mathrm{ni}+2 \mathrm{i}+1\} \\
& =\operatorname{gcd} \text { of }\{\mathrm{i},(2 \mathrm{n}+1) \mathrm{i}+1\} \\
& =1, \quad \mathrm{i}=1,2,---, \mathrm{n} \text {. } \\
& \operatorname{gcin} \text { of }\left(\mathrm{v}_{\mathrm{n}+\mathrm{i}}\right) \quad=\operatorname{gcd} \text { of }\left\{f_{\text {orpp }}^{*}\left(v_{n+i} v_{2 n+1}\right)\right. \text {, } \\
& \left.f_{\text {orpp }}^{*}\left(v_{n+i} v_{2 n+2}\right)\right\} \\
& =\operatorname{gcd} \text { of }\{(\mathrm{n}+\mathrm{i})(2 \mathrm{n}+1)+1 \text {, } \\
& (n+i)(2 n+2)+1\} \\
& =\operatorname{gcd} \text { of }\{\mathrm{n}+\mathrm{i},(\mathrm{n}+\mathrm{i})(2 \mathrm{n}+1)+1\} \\
& =1, \quad \mathrm{i}=1,2,---, \mathrm{n} \text {. } \\
& \operatorname{gcin} \text { of }\left(\mathrm{v}_{2 \mathrm{n}+1}\right) \quad=\operatorname{gcd} \text { of }\left\{f_{\text {orpp }}^{*}\left(v_{1} v_{2 n+1}\right)\right. \text {, } \\
& \left.f_{\text {orpp }}^{*}\left(v_{2} v_{2 n+1}\right)\right\} \\
& =\operatorname{gcd} \text { of }\{2 \mathrm{n}+2,4 \mathrm{n}+3\} \\
& =\operatorname{gcd} \text { of }\{2 \mathrm{n}+1,2 \mathrm{n}+2\}=1 \text {. } \\
& \operatorname{gcin} \text { of }\left(\mathrm{v}_{2 \mathrm{n}+2}\right) \quad=\operatorname{gcd} \text { of }\left\{f_{\text {orpp }}^{*}\left(v_{1} v_{2 n+2}\right)\right. \text {, } \\
& \left.f_{\text {orpp }}^{*}\left(v_{2} v_{2 n+2}\right)\right\} \\
& =\operatorname{gcd} \text { of }\{2 \mathrm{n}+3,4 \mathrm{n}+5\} \\
& =\operatorname{gcd} \text { of }\{2 n+2,2 n+3\}=1
\end{aligned}
$$

So, $g$ cin of each vertex of degree greater than one is 1 . Hence $\mathrm{G}$, admits one raised product prime labeling. 
Example 3.7 $\mathrm{G}=\mathrm{D}\left(\mathrm{K}_{1,4}\right)$

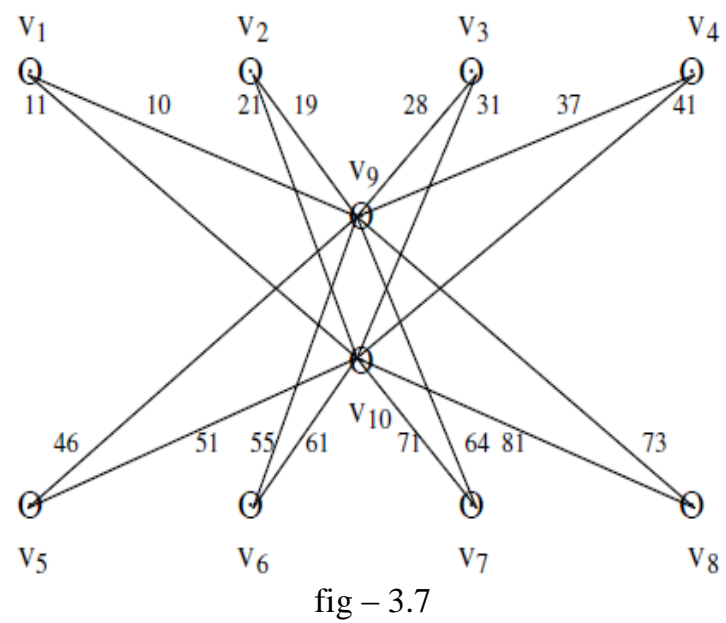

Theorem 3.8 Tensor product of path $\mathrm{P}_{2}$ and star $\mathrm{K}_{1, \mathrm{n}}$ admits one raised product prime labeling.

Proof: Let $\mathrm{G}$ be the graph and let $\mathrm{v}_{1}, \mathrm{v}_{2},---, \mathrm{v}_{2 \mathrm{n}+2}$ are the vertices of $\mathrm{G}$.

Here $|\mathrm{V}(\mathrm{G})|=2 \mathrm{n}+2$ and $|\mathrm{E}(\mathrm{G})|=2 \mathrm{n}$.

Define a function $\mathrm{f}: \mathrm{V} \rightarrow\{1,2,---, 2 \mathrm{n}+2\}$ by

$$
\mathrm{f}\left(\mathrm{v}_{\mathrm{i}}\right)=\mathrm{i}, \mathrm{i}=1,2,---, 2 \mathrm{n}+2
$$

Clearly $\mathrm{f}$ is a bijection.

For the vertex labeling $\mathrm{f}$, the induced edge labeling $f_{\text {orpp }}^{*}$ is defined as follows

$f_{\text {orpp }}^{*}\left(v_{2 n+1} v_{i}\right) \quad=\mathrm{i}(2 \mathrm{n}+1)+1, \quad \mathrm{i}=1,2,--, \mathrm{n}$.

$f_{\text {orpp }}^{*}\left(v_{2 n+2} v_{n+i}\right) \quad=(2 \mathrm{n}+2)(\mathrm{n}+\mathrm{i})+1, \mathrm{i}=1,2,---, \mathrm{n}$.

Clearly $f_{\text {orpp }}^{*}$ is an injection.

gcin of $\left(\mathrm{v}_{2 \mathrm{n}+1}\right)$

$=\operatorname{gcd}$ of $\left\{f_{\text {orpp }}^{*}\left(v_{1} v_{2 n+1}\right)\right.$,

$\left.f_{\text {orpp }}^{*}\left(v_{2} v_{2 n+1}\right)\right\}$

$=\operatorname{gcd}$ of $\{2 n+2,4 n+3\}$

$=\operatorname{gcd}$ of $\{2 \mathrm{n}+1,2 \mathrm{n}+2\}=1$.

$\operatorname{gcin}$ of $\left(\mathrm{v}_{2 \mathrm{n}+2}\right)$

$=\operatorname{gcd}$ of $\left\{f_{\text {orpp }}^{*}\left(v_{n+1} v_{2 n+2}\right)\right.$,

$\left.f_{\text {orpp }}^{*}\left(v_{n+2} v_{2 n+2}\right)\right\}$

$=\operatorname{gcd}$ of $\left\{2 n^{2}+4 n+3,2 n^{2}+6 n+5\right\}$

$=\operatorname{gcd}$ of $\{2 \mathrm{n}+2,(2 \mathrm{n}+2)(\mathrm{n}+1)+1\}$

$=1$.

So, $\boldsymbol{g c i n}$ of each vertex of degree greater than one is 1 . Hence $\mathrm{G}$, admits one raised product prime labeling.

Example 3.8 $\mathrm{G}=$ Tensor product of $\mathrm{P}_{2}$ and $\mathrm{K}_{1,3}$.

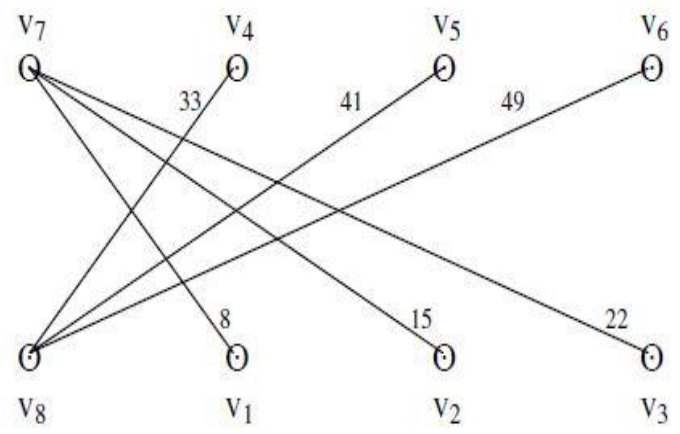

fig -3.8
Theorem 3.9 Jelly fish graph $\mathrm{JF}(\mathrm{m}, \mathrm{n})$ admits one raised product prime labeling.

Proof: Let $\mathrm{G}$ be the graph and let $\mathrm{v}_{1}, \mathrm{v}_{2},---, \mathrm{v}_{\mathrm{m}+\mathrm{n}+4}$ are the vertices of $\mathrm{G}$.

Here $|\mathrm{V}(\mathrm{G})|=\mathrm{m}+\mathrm{n}+4$ and $|\mathrm{E}(\mathrm{G})|=\mathrm{m}+\mathrm{n}+5$.

Define a function $\mathrm{f}: \mathrm{V} \rightarrow\{1,2,---, \mathrm{m}+\mathrm{n}+4\}$ by

Clearly $\mathrm{f}$ is a bijection.

$$
\mathrm{f}\left(\mathrm{v}_{\mathrm{i}}\right)=\mathrm{i}, \mathrm{i}=1,2,---\mathrm{m}+\mathrm{n}+4
$$

For the vertex labeling $\mathrm{f}$, the induced edge labeling $f_{\text {orpp }}^{*}$ is defined as follows

$f_{\text {orpp }}^{*}\left(v_{m+1} v_{i}\right) \quad=\mathrm{im}+\mathrm{i}+1, \quad \mathrm{i}=1,2,---, \mathrm{m}$.

$f_{\text {orpp }}^{*}\left(v_{m+i} v_{m+i+1}\right) \quad=(\mathrm{m}+\mathrm{i})(\mathrm{m}+\mathrm{i}+1)+1, \mathrm{i}=1,2,3$.

$f_{\text {orpp }}^{*}\left(v_{m+1} v_{m+3}\right) \quad=(\mathrm{m}+1)(\mathrm{m}+3)+1$.

$f_{\text {orpp }}^{*}\left(v_{m+2} v_{m+4}\right) \quad=(\mathrm{m}+2)(\mathrm{m}+4)+1$.

Clearly $f_{\text {orpp }}^{*}$ is an injection.

gcin of $\left(\mathrm{v}_{\mathrm{m}+\mathrm{i}}\right)$

$$
\begin{gathered}
=\operatorname{gcd} \text { of }\left\{f_{\text {orpp }}^{*}\left(v_{m+i-1} v_{m+i}\right),\right. \\
\left.\quad f_{\text {orpp }}^{*}\left(v_{m+i} v_{m+i+1}\right)\right\} \\
=\operatorname{gcd~of~}\left\{(\mathrm{m}+\mathrm{i})^{2}-(\mathrm{m}+\mathrm{i})+1,\right. \\
\left.\quad(\mathrm{m}+\mathrm{i})^{2}+(\mathrm{m}+\mathrm{i})+1\right\} \\
=\operatorname{gcd} \text { of }\{2(\mathrm{~m}+\mathrm{i}), \\
\left.\quad(\mathrm{m}+\mathrm{i})^{2}-(\mathrm{m}+\mathrm{i})+1\right\} \\
=\operatorname{gcd} \text { of }\{(\mathrm{m}+\mathrm{i}), \\
\quad(\mathrm{m}+\mathrm{i})(\mathrm{m}+\mathrm{i}-1)+1\} \\
=1, \quad \mathrm{i}=1,2,3,4 .
\end{gathered}
$$

So, gcin of each vertex of degree greater than one is 1 . Hence $\mathrm{G}$, admits one raised product prime labeling.

Example 3.9 $\mathrm{G}=\mathrm{JF}(4,3)$

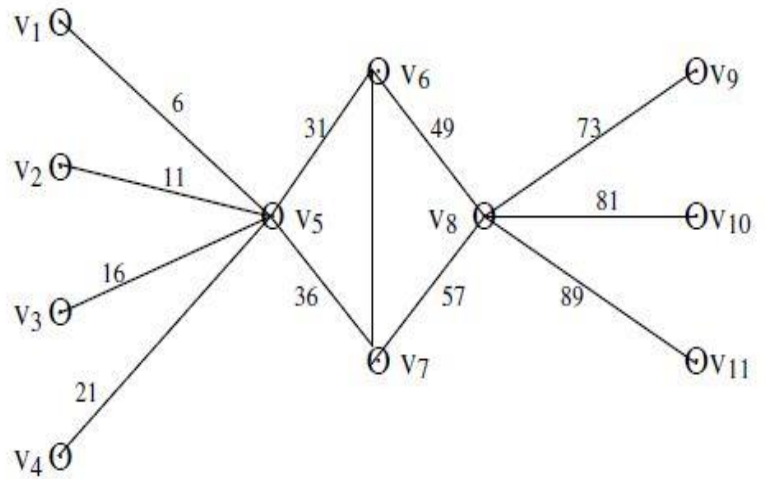

fig -2.9

\section{CONCLUSION}

Every graph is not one raised product prime graphs. So it is very interesting to find graphs or graph families which admit one raised product prime labeling. Here 9 graphs related to star graph family are investigated. This can be extended to some other graphs.

\section{REFERENCES}

[1] Apostol. Tom M, Introduction to Analytic Number Theory, Narosa, (1998).

[2] F Harary, Graph Theory, Addison-Wesley,Reading, Mass, (1972 
[3] Joseph A Gallian, A Dynamic Survey of Graph Labeling, The Electronic Journal of Combinatorics(2015), \#DS6, Pages 1-389.

[4] $\mathrm{T}$ K Mathew Varkey, Some Graph Theoretic Operations Associated with Graph Labelings, PhD Thesis, University of Kerala 2000.

[5] Sunoj B S, Mathew Varkey T K, "One Raised Product Prime Labeling of Some Cycle Related Graphs ", Journal of Harmonized Research in Applied Science, Vol.6-No.2, May 2018, pp 49-54.

[6] Sunoj B S, Mathew Varkey T K, "One Raised Product Prime Labeling of Some Snake Graphs ", International Journal of Research in Advent Technology, Vol.6-No.5, May 2018, pp 455459.

[7] Sunoj B S, Mathew Varkey T K, "One Raised Product Prime Labeling of Some Path Related Graphs", Research Guru - Online Journal of Multidisciplinary Subjects, Vol.12-No.1, June 2018, pp 950-959.

[8] Sunoj B S, Mathew Varkey T K, "Hexagonal Difference Prime Labeling of Some Path Graph”, Mapana J Sci., Vol.16, No.3, June 2017, pp 41-46.

\section{Authors Profile}

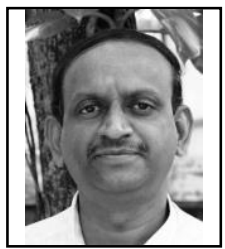

Mr. Sunoj B S pursued MSc and Mphil from Kerala University and Cochin University of Science and Technology in $1987 \& 1990$. He is currently working as Assistant Professor in Department of Mathematics from Government Polytechnic College, Attingal since 2016. He is a life member of ADMA since 2013. He has published 82 papers in reputed national and international journals.His main research work focuses on labeling and prime labeling of direct and undirect graphs. He has 25 years of teaching experience and 4 years of research experience.

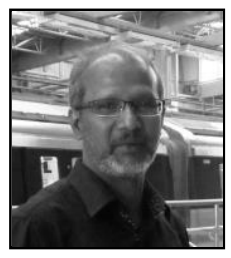

Dr.Mathew Varkey $\mathrm{T} \mathrm{K}$ pursued MSc from Mahatma Gandhi University, Kottayam, Mphil from Ramanujan Institute of Mathematical Sciences Chennai and $\mathrm{PhD}$ from Kerala University. He is currently working as Assistant Professor in Department of Mathematics T K M College of Engineering Kollam since 2004. He is a life member of KMA \& ADMA. He has published 4 text books and more than 150 papers in reputed national and international journals.His main research work focuses on labeling, fuzzy graph, semi graph and algebraic graph theory. He has 14 years of teaching experience and 10 years of research experience. He had produced one phd and three of his students submitted phd thesis. He is an editorial board member of CDSM. 